\title{
Comunicación

\section{Limitaciones y alcances del enfoque de domesticación de la tecnología en el estudio del teléfono celular}

CONSUELO YARTO WONG ${ }^{1}$

El trabajo se propone examinar la utilidad del enfoque de domesticación aplicado al estudio de las tecnologías móviles de comunicación, en general, y del teléfono celular, en particular. Con base en la revisión de la literatura, se presentan los antecedentes teóricos, se definen los conceptos relacionados con el proceso de domesticación y se profundiza en algunos supuestos básicos del mismo. Se discuten las posibilidades y limitaciones del enfoque en el estudio del celular, y se señala la necesidad de "purificar" el concepto de domesticación para ampliar la perspectiva de análisis e incluir los procesos de uso y apropiación fuera del ámbito doméstico.

PALABRAS CLAVE: domesticación, tecnología, teléfono celular, teléfono móvil, tecnologías personales de comunicación.
The purpose of this work is to examine the usefulness of the domestication approach applied to the study of mobile communication technologies in general and the cell phone in particular. Based on a review of the existing literature of the field, the work provides the background, the concepts related to the process of domestication and some basic assumptions of the theoretical framework. We discussed the possibilities and limitations of this approach in the study of cellular and noted the need to "purificate" the concept of domestication to enlarge the domain of analysis and include the processes of use and appropriation outside the domestic sphere.

KEY WORDS: domestication, technology, cell phone, mobile phone, mobile communication technologies.

1 Instituto Tecnológico y de Estudios Superiores de Monterrey. México.

Correo electrónico: cyarto@itesm.mx 


\section{INTRODUCCIÓN}

Desde su origen, el enfoque de "domesticación" estuvo ligado al estudio de las tecnologías de información y comunicación (TIC), en una perspectiva que buscaba conocer sus procesos de uso y apropiación en el ámbito doméstico, a la vez que enfatizaba el contexto social en el que se consumían, con especial atención en las rutinas, dinámicas, conflictos y negociaciones que generaban su aparición y empleo en el hogar (Silverstone \& Hirsch, 1992; Silverstone, 1994). Con ello en mente, las primeras investigaciones giraron alrededor de medios como la TV y el teléfono, dispositivos como la contestadora telefónica y el reproductor de video (Haddon \& Silverstone, 1993, 1994, 1996), tecnologías todas catalogadas como domésticas por su contexto de uso y consumo. La llegada de las consolas de videojuegos y la computadora de escritorio desplazó la atención hacia los nuevos aparatos, y puso de manifiesto las limitaciones del acercamiento para abordar los procesos de domesticación de medios que se consumían también en otros espacios como los clubes de videojuegos y las casas de los amigos, en el caso de las primeras, y los centros de trabajo o de estudio en el caso de la segunda. La aparición reciente de dispositivos móviles como el teléfono celular, los asistentes digitales personales (PDA) y las computadoras laptop, tecnologías de carácter personal diseñadas para utilizarse más allá de los límites del hogar, evidenciaron la necesidad de examinar la capacidad y eficacia del modelo para abordar los procesos de domesticación en los espacios públicos, que es donde principalmente se usan, consumen y dan sentido a estas nuevas tecnologías.

La llegada de las tecnologías móviles ha modificado de tal forma las categorías espacio-temporales, que parece evidente que se requieren conceptos y acercamientos nuevos que permitan dar cuenta de una problemática cualitativamente diferente. Sin embargo, este trabajo recurre al concepto de domesticación por considerar que, aun con sus limitaciones, resulta útil para abordar el fenómeno, adaptando algunos de sus supuestos al nuevo entorno tecnológico. Con el propósito de aportar perspectivas a la discusión, y con el teléfono celular como objeto central de interés, se plantearon las siguientes preguntas: ¿de qué manera el enfoque de domesticación resulta una perspectiva de análisis adecuada 
para el estudio de las tecnologías móviles?, ¿qué modificaciones o cambios requiere el modelo original para adaptarse al estudio del teléfono celular?, ¿qué ventajas y posibilidades ofrece para examinar las complejidades de este aparato?, ¿qué desventajas y limitaciones conlleva abordar el fenómeno a partir de los supuestos de la domesticación?

Para dar respuesta a las mismas se procedió a una revisión de la literatura que incluyó a los autores originales de la propuesta, investigadores que han seguido desarrollando este tipo de acercamiento y diversos críticos del mismo. La información obtenida se estructuró de la siguiente manera: en la primera parte se presentan los orígenes y antecedentes teóricos de la domesticación, se definen conceptos básicos y se discuten sus supuestos principales; la segunda aborda algunas adaptaciones que requiere el enfoque para estudiar las tecnologías móviles como el celular, y la última presenta las ventajas y desventajas del mismo al investigar los usos y apropiaciones de esta tecnología. Finalmente, en las conclusiones se señalan algunas adecuaciones que requiere el modelo original para aplicarse en la investigación del celular desde una perspectiva relevante y fructífera.

\section{LA DOMESTICACIÓN DE LA TECNOLOGÍA}

El enfoque de domesticación fue desarrollado originalmente por Roger Silverstone y diversos colaboradores (Silverstone, Hirsch \& Morley, 1992; Silverstone 1993, 1994; Silverstone \& Haddon, 1996) a principios de la década de 1990, e hizo coincidir tradiciones e intereses tanto del campo de la comunicación como de la sociología. Del primero asimiló los estudios etnográficos de audiencia interesados en el contexto de exposición a los medios y el papel de éstos en la vida de las personas, y del segundo el interés por el consumo y, en particular, la reflexión sobre la naturaleza simbólica de los bienes. La aparición de nuevas tecnologías de información y comunicación como los reproductores de video (VCR), la televisión satelital, las computadoras y el Internet terminaron por integrar el campo de estudio (Haddon, 2006; 2007).

Al hacer coincidir ambas perspectivas para estudiar tanto medios tradicionales como tecnologías emergentes, la domesticación se constituyó como un modelo de análisis que permitía distanciarse 
de algunas posiciones reduccionistas que centraban su interés en las motivaciones de uso desde una perspectiva individual sin considerar el contexto, como era el caso de "Usos y gratificaciones" (Katz, Blumler \& Gurevitch, 1973, 1974), y superar el determinismo tecnológico de otros enfoques que asumían la adopción de los nuevos dispositivos y medios como un proceso racional, lineal y de cierta forma tecnológicamente predeterminado, tal y como hacía "Difusión de Innovaciones" (Rogers, 1983). Este tipo de acercamiento representó también una alternativa al determinismo textual que prevalecía en algunos grupos de investigación de comunicación -particularmente británicos-, al trasladar la atención del "texto" al "contexto" (Berker, Hartmann, Punie \& Ward, 2006).

Aplicado a las TIC, el término "domesticación" es una metáfora del concepto de amansar la vida salvaje. Un proceso de doma o control que hace del objeto un miembro más del hogar: "Lo hacemos con nuestras máquinas, nuestra información, así como lo hicimos en el pasado con nuestros animales y nuestras cosechas" (Silverstone, 2004, p. 53). En sentido literal, implicaría traer desde un ámbito agreste (esfera pública) un artefacto tecnológico para subordinarlo a nuestros propios fines, encontrando para él un espacio o lugar en el ámbito doméstico (esfera privada). Pero la domesticación, señala Silverstone (1994), no trata sólo de poner las cosas bajo control, trata también de la expresión de la subjetividad de los usuarios a través de la posibilidad potencial de formar y transformar objetos y sentidos mediante el consumo y apropiación de los mismos, hasta integrarlos en la propia identidad.

El proceso involucra una serie de actividades (apropiación, objetización, incorporación, conversión) a través de las cuales una tecnología es traída desde el ámbito público donde es producida, hasta el ámbito privado donde es consumida. En el modelo original, Silverstone (et al., 1992) definen la "apropiación" como el momento en que un artefacto deja su estatus de mercancía, dentro de la economía formal, y se convierte en un objeto propiedad de alguien que, al llevarlo consigo, le confiere un significado particular. La operación refiere también a la manera como los usuarios toman conciencia de la tecnología y anticipan de qué forma y en qué situaciones ésta puede ocupar un lugar en sus vidas. Considera, además, las decisiones, consensos, conflictos y negociacio- 
nes involucrados en su adquisición (Haddon, 2003, 2006) e ingreso en un determinado espacio personal o grupal.

En la "objetización" el usuario adscribe valores cognitivos y estéticos, de manera que la tecnología adquiere un lugar y significado específico en la vida de las personas. Dichos valores se expresan tanto en el uso como en la disposición física de los aparatos, y apuntan a principios clasificatorios que definen diferencias por género y edad, así como patrones de diferenciación espacial público/privado. A través de su utilización y despliegue, el usuario se apropia de los objetos asumiendo que su consumo contribuye a la construcción y manifestación de su identidad personal (Ling, 2004), con lo que legitima su adquisición y uso (Ling \& Helmersen, 2000).

La "incorporación" refiere la manera como las tecnologías se integran en las actividades cotidianas de los sujetos de acuerdo a sus necesidades, conocimientos y preferencias, pues para ser funcional una tecnología debe poder incorporarse en las rutinas diarias y en la estructuración del tiempo de los usuarios (Ling, 1997; Silverstone, et al., 1992). Las funciones de la tecnología no están predeterminadas en su totalidad; algunas pueden cambiar o desaparecer, y los usuarios pueden resistirse a ciertos usos (Haddon, 1993, 2003) o descubrir y desarrollar algunos otros no previstos por la industria y/o el mercado.

Finalmente, durante el proceso de "conversión” la tecnología se integra en la imagen del usuario, con lo que contribuye a la definición de su capital material y simbólico, y se despliega públicamente como una forma de reafirmar cierta posición a través de su propiedad y competencia de uso. Para que la apropiación de una tecnología tenga consecuencias sociales debe ser exhibida material y simbólicamente (Silverstone, 2006; Silverstone, et al., 1992) de manera que las apreciaciones y juicios de los otros sobre el sujeto pasan a través de los aparatos que usa y posee (Ling, 2004).

Con el tiempo se adicionaron nuevas dimensiones de análisis como la de "imaginación", relacionada con la forma en que las personas toman conocimiento de una tecnología y anticipan la posición que puede ocupar en sus vidas (Haddon, 2003). En una revisión del enfoque Silverstone (2006) sustituye el concepto de apropiación por el de "como- 
dificación", que considera más preciso, y en el que incluye las labores de diseño, investigación de mercado, promoción y formación de políticas públicas que preparan el terreno para la apropiación inicial de una nueva tecnología. En la nueva propuesta, comodificación, objetización, incorporación y conversión pasan a ser dimensiones del proceso general de apropiación.

El enfoque de domesticación descansa en tres supuestos básicos: la consideración del hogar como una economía moral (Silverstone, 1991, 1994), el concepto de doble articulación de las tecnologías como objetos materiales y simbólicos (Silverstone, et al., 1992) y la idea de que las tecnologías vienen predomesticadas a partir de su proceso de diseño y comercialización (Silverstone \& Haddon, 1996).

Desde la perspectiva de domesticación, el hogar (esfera privada) se concibe como parte de un sistema transaccional de relaciones económicas y sociales involucrado activamente con la producción e intercambio de mercancías y significados provenientes de la economía social formal (esfera pública). Los intercambios, dentro de este sistema, involucran una dimensión moral en la medida que la economía doméstica opera de acuerdo a una serie de valores creados y negociados dentro de la cultura privada -"economía moral del hogar"-, valores distintos a los que operan en la economía formal que, en esos términos, constituye una economía moral diferente (Lacey, 2007).

El hogar resulta, entonces, además de una unidad económica en el sentido tradicional, una economía de significados que proveé los recursos de que disponen sus habitantes para evaluar los objetos, sujetos $\mathrm{y}$ acciones que forman la vida social, y a partir de los cuales definen y organizan sus vidas. Las posiciones morales asociadas a las prácticas cotidianas resultan fundamentales para el proyecto de "seguridad ontológica" (Giddens, 2004), y lo que está en juego en este intercambio entre lo público y lo privado es la capacidad del hogar o la familia para crear y mantener su identidad y autonomía como una unidad económica, social y cultural y, por extensión, la identidad y autonomía de sus miembros (Silverstone, et al., 1992).

En esta dinámica, en la que objetos y significados van y vienen entre la esfera pública, donde son producidos y distribuidos, y la esfera privada, donde son consumidos y apropiados, es donde mejor se evidencia 
el trabajo de reproducción social que tiene lugar en el interior de la economía moral del hogar. Y las tecnologías de información y comunicación juegan un papel particularmente relevante en este proceso por su “doble articulación” (Silverstone, 1991, 1993; Silverstone, et al., 1992) como bienes de consumo y como medios que transmiten información, comunicación y entretenimiento.

Aunque en un primer momento cualquier tecnología puede ser articulada como objeto material y como objeto simbólico, en el caso de las TIC existe una segunda articulación que opera a través de los contenidos y narrativas 2 que transmiten, lo que las convierte en mediadoras del conocimiento social y punto de encuentro entre la cultura privada y la economía formal. Las tecnologías forman parte de la economía moral del hogar como objetos materiales, pero aportan a éste la cultura pública de la que provienen, y al conectar la esfera privada con el mundo exterior, ponen a su alcance los significados compartidos por una comunidad -local, regional, nacional o mundial-, que constituyen la base para una "educación" o competencia en todos los aspectos de la cultura contemporánea (Silverstone, en Hartmann, 2006).

Además, tanto en el consumo de sí mismas como mercancías, como a través del consumo de los contenidos que ofrecen, tecnologías y medios promueven prácticas y modelos de uso y apropiación mediante los cuales se expresan y construyen identidades -individuales y colectivas- y definiciones de poder y estatus por género y por edad. Medios y tecnologías constituyen elementos fundamentales para nuestro "ser en el mundo" como consumidores, ciudadanos, individuos o miembros de una familia (Hartmann, 2006), por su capacidad para ayudar al individuo y a la colectividad a definir su identidad y sostener su seguridad ontológica (Silverstone, 2006).

Desde este enfoque, las tecnologías son tanto utilitarias como simbólicas (Haddon, 1998; Katz \& Aakhus, 2002) y su domesticación va más allá de comprar y usar un aparato: involucra las decisiones y esfuerzos

2 La segunda articulación considera los programas individuales, los géneros reconocidos por las audiencias, los horarios de programación, lo que se dice acerca del medio y sus contenidos, los patrones de atención y, en general, su uso en la vida diaria. 
requeridos para la adquisición del mismo, los acuerdos sobre el lugar que deberá ocupar en el espacio y tiempo doméstico y, en general, su papel en la vida diaria de los miembros del hogar. Su adopción genera políticas de uso que regulan el acceso y disposición de las mismas y da lugar a procesos de negociación y lucha para mantener, ganar o recuperar su control (Silverstone, 1994), de tal suerte que su presencia abre posibilidades que modifican no sólo las prácticas rutinarias del hogar, sino también la relación entre sus miembros (Morley, 1992), cómo construyen sus redes de distinción y pretensiones de identidad (Morley \& Silverstone, 1990), la adquisición de competencias culturales (Morley \& Silverstone, 1990; Silverstone, 2002), e incluso la forma de conceptualizar el tiempo y espacio sociocultural (Haddon, 1993; Silverstone, 2004).

Una última consideración relevante del modelo alude al papel de la industria y el mercado en el proceso, a través de la "predomesticación" de la tecnología. Tecnologías y medios de comunicación nunca son ingenuos: vienen preformados con atribuciones y sentidos que se establecen a partir de su proceso de diseño, mercadotecnia y comercialización, a los que luego se suman aquellos que emergen del discurso social que genera su aparición, más los que finalmente les adjudican usuarios y consumidores una vez que los adquieren e integran en sus actividades cotidianas. Para Silverstone y Haddon el uso y apropiación de una tecnología están anticipados en su proceso de diseño, aunque el ciclo sólo se completa durante la domesticación por parte del sujeto, design/domestication interface. Ambos "dependen de un particular balance entre estructura y agencia, en el cual los procesos institucionales -económico, político y cultural- posibilitan, al mismo tiempo que restringen, la capacidad de los consumidores para definir su propia relación con la tecnología" (1996, p. 46).

Las constricciones de la industria y del mercado son invisibles para la mayoría de los consumidores y están integradas tanto en el diseño y promoción de la tecnología como en las definiciones públicas de cómo y para qué deberá ser usada. Pero tales definiciones no proceden únicamente de estructuras regulatorias, manuales de uso o discursos publicitarios, derivan también de las rutinas cotidianas en las que participa el sujeto $y$, particularmente, de las interacciones que establece con otros tanto en el seno de la familia (pareja, hijos, padres), como fuera de ella 
(amigos, compañeros, colegas de trabajo), quienes de cierta manera delimitan los usos posibles de la tecnología y definen en parte sus consecuencias (Haddon, 1993, 1994). En esa dirección parece inclinarse Lasen (2005) cuando destaca la importancia de considerar la adopción de los dispositivos tecnológicos como un conjunto de prácticas compartidas dentro de diferentes grupos, que encuentran distintas formas de emplear los aparatos. De esta manera, aunque todos los objetos de consumo son producto de intereses comerciales y procesos industriales, diferentes grupos pueden entablar distintos tipos de vinculación con los mismos, subrayando el aspecto relacional del fenómeno de domesticación y la necesidad imprescindible de analizar el contexto de uso.

\section{LA DOMESTICACIÓN DE LAS TECNOLOGÍAS MÓVILES:}

\section{ADAPTACIONES AL ENFOQUE}

Aunque inicialmente los estudios de domesticación se enfocaron al consumo de tecnologías en el ámbito doméstico (teléfono, televisión, computadora familiar), la posibilidad de acceso a las TIC desde distintos lugares (escuelas, centros de trabajo, cibercafés) y la aparición y comercialización masiva de dispositivos móviles (laptops, PDA y teléfonos celulares) han obligado a ampliar la perspectiva tanto para considerar las prácticas de consumo de las tecnologías en otros "espacios" de la vida diaria (Haddon, 1998, 2000, 2003), como para incluir nuevas dimensiones de análisis en el estudio de las mismas (Haddon, 2006).

Helle-Valle y Slettemeås (2008) destacan la necesidad de "purificar" el concepto de domesticación disociándolo de su relación con lo privado y lo doméstico, tanto para eliminar la ambigüedad con que el término es utilizado, ${ }^{3}$ como para evitar las limitaciones que esto supone a la investigación. En su opinión, la asociación exclusiva del término con el acto de domesticar permite conceptualizar el fenómeno como un proceso de aculturación, ${ }^{4}$ que tiene lugar tanto dentro como fuera

3 En ocasiones se refiere al hecho de traer un objeto dentro de la esfera doméstica y en otras a la acción de domesticar lo salvaje.

4 Ya en el prólogo de Consuming Technologies. Media and Information in Domestic Spaces, considerado texto seminal dentro del enfoque, Strathern 
del hogar, que brinda mayor poder explicativo al extender su alcance a contextos no domésticos y posibilita su aplicación a cualquier medio o tecnología, incluidos aquellos en los que el proceso de domesticación involucra los ámbitos público y privado.

Bakardjieva (2006) señala, por su parte, que las condiciones que sustentaban algunos de los supuestos originales del modelo han cambiado tan radicalmente que demandan ciertas adaptaciones del mismo. En la sociedad actual resulta difícil hablar de un "orden moral" estable (“economía moral") porque los valores y estilos de vida de los hogares y las familias son renegociados continuamente; la separación entre lo público y lo privado es cada vez más imprecisa en la medida en que actividades de trabajo invaden el hogar y conversaciones personales llenan los espacios públicos, y las tecnologías producidas por la industria y distribuidas por el mercado son vistas más como herramientas que como bienes de consumo. Para que la perspectiva de domesticación siga siendo útil en este nuevo contexto, propone sustituir el concepto de "hogar" por el de "casa" y el de "consumo" por el de "vida diaria".

Desde esta posición, la "casa" sería, más que un lugar, una experiencia fenomenológica asociada a un sentimiento de seguridad, confianza, libertad y control sobre los propios asuntos; la contenedora de las relaciones interpersonales que sustentan el proyecto identitario, a través del cual el sujeto como "agente" revisa sus estrategias de acción para operar de cara a las fuerzas opresivas de la "estructura". Esta conceptualización permite superar las limitaciones de una noción estática del hogar al considerar la dinámica constante de las relaciones entre lo público y lo privado y entre la agencia y la estructura. En cuanto a la noción tradicional de consumo, al concentrar la atención en el aspecto económico de la producción, hace aparecer al primero como un proceso subordinado. Y la domesticación es consumo desde el punto de vista de los diseñadores, productores y el mercado, pero desde la perspectiva de los usuarios es parte de la vida diaria. Y la "vida diaria", como categoría analítica, es más comprehensiva que "consumo" en la medida que

(1992) conceptualiza la domesticación como un proceso de enculturación, aunque enfocado primordialmente al ámbito familiar por ser el contexto en que se consumían las tecnologías estudiadas. 
considera un tipo diferente de producción: la de nuevos sentidos por parte de los sujetos al momento que usan y se apropian de los objetos, aspecto fundamental en la construcción de su vida cotidiana.

En esa misma línea, la perspectiva escandinava de los estudios de domesticación de la tecnología visualiza el proceso como una serie de actividades relacionadas con la construcción de la vida diaria más que con el consumo en el hogar. La domesticación involucra la creación de un conjunto de prácticas asociadas con un determinado artefacto tecnológico, la elaboración de los significados del mismo -incluido su papel en la producción de la identidad de los usuarios-, y los procesos cognitivos relacionados tanto con su aprendizaje y práctica como con la producción de nuevos significados. Todas estas actividades aparecen vinculadas con los distintos lugares donde se usan y consumen las tecnologías, de manera que su domesticación resulta un proceso multisituado que trasciende el hogar (Sørensen, 2006), dando cabida al análisis de las mismas tanto en los espacios privados como fuera de ellos.

Haddon (2006), por su parte, subraya la utilidad de complementar este marco de aproximación con otras formas o niveles de análisis, y sugiere incorporar las biografías de los sujetos para analizar los cambios en el uso y apropiación de medios y tecnologías en diferentes etapas o circunstancias de sus vidas; abordar grupos generacionales con experiencias compartidas que hayan moldeado sus percepciones, preferencias, hábitos y valores relacionados con el uso de las TIC; o seguir la carrera de una tecnología y los cambios que presenta a medida que incorpora nuevas funciones o se recombina con otros medios. Todo ello con el propósito de develar las particularidades de cada tecnología y la complejidad de los nuevos contextos de uso.

De esta manera, ampliar el enfoque para considerar el proceso de domesticación de las tecnologías no sólo desde la perspectiva doméstica sino desde otros espacios, entender el consumo de las mismas como un proceso ligado íntimamente a la producción de sentido en el contexto de la vida diaria, e incluir nuevas dimensiones que permitan una aproximación más exhaustiva al fenómeno, otorga mayor capacidad de análisis al modelo, particularmente cuando se aplica el estudio de las tecnologías personales móviles de comunicación, como el teléfono celular. 


\section{LA DOMESTICACIÓN DEL TELÉFONO CELULAR}

El enfoque original de domesticación resulta útil para formular preguntas relacionadas con los procesos iniciales de adopción del celular, así como las justificaciones para apoyar decisiones de compra, las políticas domésticas de acceso y uso, la apropiación individual por parte de los sujetos y la manera como su presencia modifica las rutinas de la casa y la relación entre quienes la integran. Sin embargo, por tratarse de una tecnología diseñada para utilizarse primordialmente más allá de los límites del hogar, ${ }^{5}$ se evidencia la necesidad de considerar también el papel que juegan amigos, compañeros de trabajo y demás redes sociales en las que interactúan los usuarios al momento de establecer prácticas de uso. Y aunque la familia sigue ejerciendo una influencia determinante sobre el consumo tecnológico de sus miembros, los procesos personales y familiares de domesticación se insertan en un proceso más amplio de domesticación social (Castells, Fernandez-Ardevol, Linchuan \& Sey, 2007).

$\mathrm{Al}$ abordar el estudio del celular desde esta perspectiva encontramos que, al igual que sucede con otras tecnologías, su domesticación responde a una economía moral que combina significados de la esfera privada y la pública a partir de los cuales se generan una serie de prácticas, políticas de uso y nuevos significados asociados al mismo. Pero a diferencia de lo que sucede con las tecnologías eminentemente domésticas, donde las normas que regulan las prácticas de uso y apropiación se construyen dentro del hogar - politics of living room-, 6 en el caso del teléfono celular se construyen tanto en la esfera privada como en la pública a partir de procesos de interacción entre los miembros de la casa y a través de los intercambios que se establecen con otras redes sociales fuera de la

5 La situación al parecer se está modificando ante el creciente número de hogares, particularmente de parejas jóvenes, que prefieren contratar servicios de telefonía celular para cada miembro en lugar de una línea doméstica (Fortunati \& Magnanelli, 2002), o familias de escasos recursos en los que un celular es el teléfono de la casa -y de todos los miembros de la familia-, ante la imposibilidad de acceder a una línea fija.

6 Morley (1992) introduce el concepto politics of living room, acuñado por Cubitt, en sus estudios sobre el consumo de TV en los hogares británicos. 
misma (Ling, 2008). Es por ello que el teléfono móvil no sólo transforma y reordena las relaciones entre los sujetos (Gesser, 2005; Licoppe \& Heurtin, 2002; Ling \& Ytrri, 2002), sino también las relaciones de estos con los espacios que ocupan (Höflich, 2004; Humphreys, 2005), desdibujando la frontera entre lo privado y lo público (Höflich, 2005), lo personal y lo social, de manera que, como apunta Morley (1992), innovaciones tecnológicas, relaciones sociales e identidades culturales quedan íntimamente ligadas.

Sørensen (2006) sugiere que en el caso de las tecnologías personales de comunicación, como el teléfono móvil, el proceso de domesticación involucra un tipo de "economía moral personal" sustentada en un particular sentido del yo y en una serie de conductas y principios morales que los usuarios -en especial los jóvenes- consideran correctos. Esta moralidad personal orienta cierto tipo de empleo y valoración del celular que, cuando es compartida por el grupo de pares, da origen al surgimiento de culturas particulares como la identificada por Castells (et al., 2007) en relación con el uso del celular entre los jóvenes a nivel mundial.

Como el resto de las tecnologías de comunicación, el teléfono celular se presenta también como doblemente articulado: por un lado es un objeto de consumo que se adquiere por motivos tanto funcionales como estéticos, y por el otro un medio de comunicación que nos conecta con el mundo y con los demás, al mismo tiempo que filtra y delimita nuestros contactos con ambos. Esta segunda articulación resulta particularmente compleja en una tecnología que opera como medio de comunicación interpersonal a través de llamadas y mensajes de texto dirigidos a un grupo de conocidos más o menos cercanos, y como medio de comunicación masiva mediante la difusión pública de contenidos informativos y de entretenimiento a través de su conexión a Internet. Aunque son estos últimos -los mensajes públicos- los asociados a una segunda articulación, no puede desestimarse el papel que juegan los mensajes privados en la transmisión de ideas, opiniones y valores que derivan en la aparición de nuevas prácticas sociales -en el caso del celular nuevos modos de comunicación, coordinación y control- $\mathrm{o}$ en la construcción de la identidad personal.

En esa línea se ubica la propuesta de Hartmann (2006) de incorporar al análisis una "triple articulación" que permita ir más allá de la naturaleza ritual del uso de los medios o la idea de participación en 
una cultura pública, para abordar las instancias de comunicación individual que ofrecen las nuevas tecnologías. Aunque el autor reconoce que es el fenómeno de "mediatización" en su conjunto -más que los mensajes individuales-, el que ejerce mayor impacto en la transformación de la vida social, subraya la importancia de estudiar las nuevas prácticas de comunicación que posibilitan dichas tecnologías y los contenidos asociados a ellas -la llamada personal o el mensaje de texto en el caso del celular, o los correos electrónicos o el bookmark en el caso de las computadoras con acceso a Internet-, como una forma de asociar el análisis del texto al del contexto. El rápido crecimiento del mercado de dispositivos personales de comunicación y la tendencia creciente al consumo de contenidos personalizados parecen justificar la propuesta. ${ }^{7}$

En análisis posteriores, Silverstone propone utilizar el concepto de doble articulación para contrastar el análisis de los medios y las tecnologías como objetos materiales con el análisis de los mismos como textos o mensajes simbólicos, abordando de manera más directa cuestiones relacionadas con la domesticación de tecnologías personales como el teléfono celular; adicionalmente, el término resulta de gran utilidad para acercarse al público/usuario de los medios/tecnologías en su doble articulación: como consumidor-espectador para medios tradicionales y como consumidor-usuario para las nuevas tecnologías (Livingstone, 2007).

Finalmente, aunque el teléfono celular viene predomesticado con una serie de funciones y sentidos asignados por la industria, se presenta como la tecnología de comunicación que mayores posibilidades ofrece para un uso y apropiación que escapan a las determinaciones de su diseño y comercialización. Prueba de ello es el impulso no previsto del

7 Sin mostrarse entusiasta con la idea, Silverstone (2006) tampoco rechaza la propuesta de explorar esta tercera articulación, aunque subraya las dificultades epistemológicas y metodológicas que ello supone, particularmente al estudiar los nuevos dispositivos personales móviles de comunicación. Destaca en especial el compromiso que requiere la aproximación etnográfica y el análisis de los textos y significados, tanto en su producción como en su consumo en el hogar, y su articulación con la esfera pública. 
envío de mensajes de texto (SMS) ${ }^{8}$ por sobre las llamadas de voz en el segmento de usuarios jóvenes y adolescentes, o su uso creativo del teléfono móvil como un instrumento para comunicarse sin pagar a través de las "llamadas bomba". 9 Son ellos, también, quienes han convertido al celular en un objeto de entretenimiento, alentando a la industria a ofrecer aparatos con juegos, cámaras fotográficas y reproductores de música y video.

Adicionalmente, y sin menoscabo del papel de la industria en la estandarización de ciertos usos y significados asociados al celular, podría suponerse que al ser ésta una tecnología eminentemente personal-que se apropia de manera individual-, las modalidades de domesticación sean más diversificadas y heterogéneas derivando en un proceso de co-construcción del teléfono móvil, en un continuo rediseño por parte de los compradores-usuarios-consumidores, quienes presionan a la industria para que modifique no sólo los aspectos físicos del aparato, sino también las funciones y servicios que ofrece de manera que satisfagan mejor sus necesidades y expectativas (Fortunati, 2006). ${ }^{10}$

Lo señalado hasta ahora apunta a que, en general, el enfoque de domesticación resulta una perspectiva adecuada y útil para analizar cómo el celular se integra en la vida diaria de los usuarios. Sin embargo, dada la complejidad de este medio, debido a su propia naturaleza e innovación, en el siguiente apartado se discuten con más detalle las ventajas y desventajas de la domesticación como perspectiva teórico-metodológica para acercarse al estudio del teléfono móvil.

8 Originalmente, el servicio de SMS (short message system) fue dirigido al segmento de hombres de negocios, quienes lo rechazaron por considerarlo poco práctico.

9 Las "llamadas bomba" son llamadas que no se pretende que sean contestadas por el destinatario para evitar el costo económico de las mismas, pero que en sí mismas tiene un significado acordado de manera previa entre los usuarios.

10 Este proceso de co-construcción no resulta privativo del celular y más bien parece ser una tendencia entre los nuevos medios y tecnologías de comunicación y los productos asociados a ellos, como los servicios digitales personalizados de información y entretenimiento. 


\section{VENTAJAS Y DESVENTAJAS}

\section{DEL ENFOQUE DE DOMESTICACIÓN}

\section{APLICADO AL ESTUDIO DEL CELULAR}

Las principales ventajas que ofrece el enfoque de domesticación parecen desprenderse de sus propias características y particularidades. Haddon (2003) identifica ciertos atributos que sirven como punto de partida para explorar las potencialidades que brinda esta perspectiva de análisis al estudio de las TIC en general, y del teléfono celular en particular. El autor señala como primer rasgo distintivo del enfoque su “énfasis en el consumo y no sólo en el uso", lo que posibilita abordar de manera amplia las dimensiones simbólicas de la tecnología. La domesticación no se interesa únicamente por la manera en que las personas utilizan el celular, se plantea también aspectos relacionadas con el tipo de vida e identidad al que aspiran los usuarios a partir de lo que dicen del aparato, la forma como lo exhiben y las estrategias individuales y familiares de control, tanto en el sentido de regular cómo, cuándo y cuánto hablan los otros, como el papel del dispositivo en las actividades personales.

En segundo lugar, el acercamiento entiende "la domesticación como un proceso sujeto a cambios y negociaciones en el que los objetos son definidos y redefinidos culturalmente a partir de su uso y en la interacción con los otros". Este punto, en particular, pone en evidencia la relación entre tecnología y cambio social, en la medida que la domesticación de un dispositivo tecnológico aparece asociada a una serie de actividades y rutinas que se crean, modifican y/o desaparecen en el uso cotidiano; el seguimiento de dichas actividades posibilita delinear la "biografía" del aparato, develando múltiples aspectos de la sociedad en la que este se localiza y consume. El estudio de la domesticación del celular permite identificar nuevas maneras en que la gente se relaciona, divierte, consume, enamora, trabaja y participa en actividades políticas y religiosas, así como los nuevos significados sociales adheridos al mismo como medio de control, independencia, seguridad o estatus.

En tercero, "la domesticación no conceptualiza el proceso de adopción como un ciclo que necesariamente debe ser completado": no hay que asumir, señala Haddon, que la domesticación es siempre exitosa. 
Un proceso de adopción puede ser reevaluado e interrumpido porque el usuario no se sintió a gusto con la tecnología, entró en una etapa diferente de actividades o consideró que ésta no va de acuerdo con su estilo de vida, de manera que habrá que contemplar también procesos de redomesticación e incluso de desdomesticación (Morley, 2006). En el caso del celular, la apropiación del aparato puede generar sentimientos ambivalentes, de manera que un usuario puede desear controlar a otros a través del aparato pero resistirse a que lo controlen, o considerarlo como necesario pero sentirse incómodo de incorporarlo a su imagen personal; algunos más pueden decidir usarlo sólo para emergencias y otros desecharlo por no sentirse aptos en el manejo de la tecnología. La perspectiva de domesticación permite abordar todas estas variantes, $\mathrm{y}$ adicionalmente servir como punto de partida para analizar las decisiones de quienes no desean adoptar la tecnología.

Una cuarta característica del modelo es su "atención al contexto de uso de las tecnologías y particularmente al papel de los otros -incluidos los no usuarios- en la experiencia que se tiene de las mismas". La importancia de este punto es que permite traer a discusión aspectos relacionados tanto con cuestiones de poder y subordinación en el hogar - especialmente en términos de género y edad-, como de normas y políticas en los espacios públicos. En el ámbito doméstico, el uso que hacen los jóvenes del celular y las estrategias de independencia que desarrollan deben enfrentar las políticas establecidas por los padres, mientras que en el ámbito público el empleo del mismo, en sitios comunes, se enfrenta a la aprobación o rechazo tanto de usuarios como no usuarios a partir de reglas poco claras y que varían de cultura a cultura.

Finalmente, la domesticación supone que la forma como se experimenta el significado de la tecnología moldea la experiencia del resto de las prácticas sociales del usuario y, a su vez, es moldeada por éstas, de manera que "el uso y apropiación de la tecnología no está determinada completamente por su funcionalidad técnica o las representaciones sociales que la rodean, sino que es estructurada también por el contexto social de los usuarios/consumidores". La ventaja que ofrece este supuesto es que permite superar los enfoques reduccionistas del determinismo tecnológico y del social, facilitando un acercamiento tanto desde la perspectiva de la industria (predomesticación) como desde la 
perspectiva de los usuarios (domesticación), lo que en el caso del teléfono celular resulta imprescindible dado el papel tan activo que juegan al impulsar usos y prácticas no previstas por el mercado.

La perspectiva de domesticación, sin embargo, no ha permanecido exenta de cuestionamientos y críticas. Un primer señalamiento refiere a la naturaleza misma del enfoque que, al centrarse en los procesos de uso y apropiación de las TIC en el ámbito doméstico, deja de lado las particularidades de su domesticación en los espacios públicos. Es evidente que en el caso de las tecnologías móviles la perspectiva original es insuficiente para dar cuenta de las prácticas de uso y de sus repercusiones sociales, por lo que resulta indispensable ampliar el alcance del análisis más allá del ámbito doméstico para incluir los otros espacios en los que los sujetos desarrollan sus actividades cotidianas.

Otro cuestionamiento básico apunta a la falta de claridad lógica del enfoque (Katz \& Sugiyama, 2006), y a eso parece referirse Ling (2001) cuando señala que la secuencia del proceso de domesticación no sigue necesariamente una progresión lineal, como parece desprenderse del modelo. El camino que va desde imaginar el papel que la tecnología puede tener en la vida de uno, adquirirla, llevarla al espacio personal, adjudicarle un sentido e integrarla en las actividades cotidianas hasta el punto de convertirla en parte de uno mismo aparece, a nivel teórico, como un proceso secuencial. En la vida cotidiana, sin embargo, no siempre sucede de esta manera. Ciertas operaciones se cumplen sin que se haya completado la anterior o dos fases pueden tener lugar al mismo tiempo. En el ámbito de las TIC, donde innovaciones y mejoras son continuas, el proceso de domesticación puede requerir ajustes a medida que se desarrolla, y particularmente en el caso del celular, donde los cambios se presentan día con día: cada nueva función que se incorpora, cada nuevo modelo que sale al mercado, cada nuevo significado que se le adhiere pueden reorientar el proceso de manera substancial, lo que impulsa a conceptualizar la domesticación como algo siempre en marcha y no necesariamente con un final exitoso.

Katz y Sugiyama (2006), aunque destacan como positivo el interés del enfoque en la naturaleza simbólica de las nuevas tecnologías, señalan la insuficiencia de éste para dar cuenta por sí solo del importante papel que juega el celular en la vida de las personas como objeto de 
moda y símbolo de estatus. El teléfono móvil, apuntan, no desaparece de la esfera pública una vez que es domesticado. Por el contrario, se afianza y evoluciona cada vez más como un recurso sobre el que se crea y expresa la identidad social, y de ahí la necesidad de ir más allá para apreciar su papel como objeto simbólico. Cabe recordar que, en su origen, el enfoque se aplicó a tecnologías que se consideraban aparatos del hogar más que propiedad de alguien, mientras que el celular es un medio de comunicación personal que se lleva encima todo el tiempo, de manera que fácilmente puede integrarse con la imagen del usuario, que en ocasiones lo exhibe para expresar su estatus o su sentido de la moda. La aparición de los dispositivos personales de comunicación, más orientados al individuo y a sus gustos particulares, obliga a una reconsideración para traer al campo aspectos relacionados con el uso de los mismos desde una perspectiva eminentemente estética.

Por otra parte, aunque la domesticación resulta un acercamiento adecuado para estudiar procesos de uso y apropiación de tecnologías que se presentan como novedosas e importantes, pierde mucho de su poder analítico al aplicarse a objetos comunes que se han integrado en las rutinas cotidianas del usuario (Ling, 2004). En el caso particular del celular, al ser una tecnología relativamente nueva para grandes sectores de población, el enfoque resulta una herramienta de análisis apropiada. Sin embargo, a medida que vaya perdiendo su "aura" de novedad y se convierte en una tecnología más, el concepto de domesticación perderá también gran parte de su poder explicativo y habrá que buscar otras perspectivas teóricas para profundizar en el estudio de este medio de comunicación.

Del lado de la metodología surgen dos limitaciones adicionales: la falta de capacidad del modelo para anticipar el patrón de adopción -prognosis- de una tecnología o medio de comunicación, dado que no ofrece una teoría formal que brinde hipótesis que puedan ser probadas, por lo que debe considerársele como una aproximación de investigación más que como una herramienta de predicción (Katz, et al., 2002; Ling, 2001, 2004), y las restricciones que supone un análisis de nivel microsocial que, adicionalmente, no establece la manera como los fenómenos particulares de domesticación se insertan en el tejido social (Bakardjieva, 2006). 
La primera acusación pudiera parecer injustificada dado que, en general, las teorías en el campo de los estudios sociales tienden a ser más explicativas que predictivas, a diferencia de lo que sucede en el campo de las ciencias exactas. El mismo Silverstone destaca que la domesticación, más que una teoría formal per se, es sobre todo una "manera de pensar" (2006, p. 229) acerca de la incorporación de la tecnología en la vida diaria, y como tal privilegia una forma de acercarse al fenómeno por sobre otro tipo de abordajes.

En cuanto a la segunda crítica, el autor señala que la domesticación conecta a priori lo macro y lo microsocial, de manera que las prácticas de uso, los procesos de propiedad y control, y las relaciones y negociaciones que se observan empíricamente en el seno del hogar son fundamentalmente dependientes de la yuxtaposición de lo público y lo privado y de su continua negociación. Además, como señala Sørensen: "El conflicto social, la disciplina y el poder son inherentes a la mayoría de las prácticas de domesticación" (2006, p. 48), de manera que los procesos dentro del hogar son un modelo del enfrentamiento entre estructura y agencia que se reproduce luego a nivel macrosocial.

Dado lo anterior, el principal reto que enfrentan los estudios de domesticación es identificar la importancia de las TIC en el ámbito individual o familiar para posteriormente determinar su impacto en el ámbito social, y a partir de ahí proponer políticas que promuevan no sólo el acceso a las mismas para los sectores marginados, sino, además, esquemas de uso que les permitan mejorar su calidad de vida y su desarrollo personal.

\section{CONCLUSIONES}

Sin perder de vista las limitaciones señaladas, la domesticación de la tecnología resulta un marco teórico pertinente y valioso para estudiar los procesos de uso y significación de dispositivos de comunicación personal como el teléfono celular. Su flexibilidad y amplitud de intereses permite adaptar la perspectiva para abordar la domesticación de las nuevas tecnologías móviles, tanto en el ámbito público como en el privado, proporcionando una visión amplia y diversificada del fenómeno.

En el caso de tecnologías relativamente nuevas o poco estudiadas, como el teléfono móvil -que hasta hace poco tiempo se convirtió en 
objeto relevante de investigación, particularmente en los países menos desarrollados-, un primer acercamiento como el que ofrece este enfoque facilita al investigador sensibilizarse sobre numerosos aspectos relacionados con el uso del aparato, pero también con las motivaciones e intereses para adquirirlo, las negociaciones y justificaciones involucradas en la compra del mismo, su ingreso y acomodo en el espacio privado del usuario, los valores utilitarios y simbólicos que éste le asigna, su integración en las actividades cotidianas y el impacto sobre las mismas, e incluso como se exhibe ante los demás para crear una identidad o proyectar una imagen. Esta diversidad de temas se puede abordar al mismo tiempo a partir de un único enfoque, lo que convierte a la domesticación en un acercamiento sumamente fructífero.

Además, al ser una perspectiva de nivel micro, centrado en la vida cotidiana de las personas, la domesticación se ajusta convenientemente a los supuestos de una aproximación cualitativa - tan validada en la investigación de las nuevas tecnologías de comunicación- otorgando preeminencia a las prácticas diarias del sujeto y a la manera en que éste les otorga sentido. Particularmente en el caso del celular, el estudio del mismo pasa inevitablemente a través de las rutinas cotidianas de los usuarios a las que se ha venido incorporado de forma sutil pero ininterrumpida. Esta incorporación involucra una dimensión simbólica - entendida como la producción/consumo de sentido- que reviste al aparato con nuevos significados sociales construidos a partir de la interacción con los otros, de modo que el dispositivo tecnológico pasa a ser, además de medio de comunicación, una herramienta de trabajo, un instrumento de control o independencia -según convenga-, un elemento de seguridad o un símbolo de estatus. El énfasis de la domesticación en los procesos de significación contribuye a esclarecer la complejidad del proceso de coconstrucción del celular como objeto cultural, aspecto fundamental para identificar las diferencias de apropiación entre individuos, grupos y culturas.

De la revisión de la literatura se desprende que utilizar el enfoque de domesticación para estudiar el teléfono celular requiere una serie de adecuaciones que permitan ampliar su alcance geográfico y adicionar nuevas dimensiones de análisis, lo que supera, de esta manera, las limitantes que conlleva su interés original, centrado en los procesos de domesticación al interior del hogar. Las adecuaciones incluyen, en primer lugar, 
que se considere la domesticación como un proceso de aculturación que tiene lugar tanto en el interior del hogar como fuera de él; segundo, que la atención sobre cómo la tecnología reconfigura las interacciones de los miembros en el hogar se amplíe para incluir las relaciones con amigos, compañeros de trabajo, familiares lejanos y todos aquellos con los que se establece comunicación a través del celular; tercero, que al análisis de las políticas domésticas de uso se incorpore el de la etiqueta social, que prescribe el empleo y disposición correcta del dispositivo en espacios públicos como restaurantes, salas de espectáculos o medios de transporte; cuarto, que a los sentidos dados a la tecnología a nivel familiar se sumen los significados públicos, que surgen alrededor del móvil como herramienta de seguridad, medio de control o símbolo de estatus, más los que le asigna el usuario al incorporarlo como parte de su imagen; quinto, que el despliegue y exhibición del dispositivo tecnológico como una afirmación de los valores cognitivos y estéticos de la familia o del hogar se considere, a nivel individual, como una declaración de identidad del usuario; y por último, que la perspectiva del consumidor-espectador de los medios tradicionales se complemente con la del consumidor-usuario para las nuevas tecnologías. Con estas modificaciones, los aspectos que permiten abordar el fenómeno a nivel doméstico sirven también de punto de partida para analizar la domesticación en un contexto de mayor alcance geográfico-social.

Sin embargo, dada la diversidad y complejidad de los fenómenos de comunicación asociados a las tecnologías móviles, empezar a desarrollar nuevas aproximaciones teóricas y metodológicas que permitan rebasar los supuestos de la domesticación para profundizar en la problemática del papel de las TIC en la sociedad contemporánea, pareciera una labor ineludible para los investigadores del campo. Y aunque el enfoque de domesticación representa un buen punto de partida y una perspectiva de análisis fructífera, se hace evidente la necesidad de ir más allá y desarrollar un concepto más específico y acorde a este nuevo objeto de estudio.

Por último, hay que señalar que, aunque el enfoque de domesticación tiene su propia agenda, es necesario empezar a cuestionarse por el tipo de investigación que demandará el estudio del teléfono móvil una vez que éste pierda su aura de novedad y se convierta en una tecnología más. En algunos años la mayoría de los usuarios serán nativos 
de esta tecnología, los usuarios jóvenes actuales se habrán convertido en los usuarios adultos, y más países -incluidas algunas naciones en desarrollo- alcanzarán la saturación del mercado. Todo ello, más la evolución que está teniendo el aparato convirtiéndose en un nuevo y poderoso dispositivo tecnológico que integra funcionalidades de distintos medios, obligarán a revisar los hallazgos y conclusiones de estudios previos para replantear preguntas e hipótesis de investigación buscando profundizar en el impacto social de un aparato cada vez más pequeño, pero cada vez más poderoso.

\section{Bibliografía}

Bakardjieva, M. (2006). Domestication running wild. From the moral economy of the household to the mores of a culture. En T. Berker, M. Hartmann, Y. Punie \& K. Ward (Eds.), Domestication of media and technology (pp. 62-79). Berkshire: Open University Press.

Berker, T., Hartmann, M., Punie Y. \& Ward, K. (2006). Introduction. En T. Berker, M. Hartmann, Y. Punie \& K. Ward (Eds.), Domestication of media and technology (pp.1-17). Berkshire: Open University Press.

Castells, M., Fernández-Ardevol, M., Linchuan, J. \& Sey, A. (2007). Mobile communication and society. A global perspective. Cambridge: The MIT Press.

Fortunati, L. (2002). Italy: stereotypes, true and false. En J. E. Katz \& M. Aakhus (Eds.), Perpetual contact: Mobile communication, private talk, public performance (pp. 42-62). Cambridge UK: Cambridge University Press.

Fortunati, L. \& Magnanelli, A. (2002). Young people and the mobile telephone. Revista de Estudios de Juventud, 57, 59-78. Recuperado en abril 15 de 2007 de la página de la Unión Internacional de Telecomunicaciones.

Fortunati, L. (2006). User Design and the Democratization of the Mobile Phone. First Monday, 11 (7). Recuperado en septiembre 8 de 2008 de la página de la publicación FirstMonday.

Gesser, H. (2005). Is the cell phone undermining the social order? Understanding mobile technology from a sociological perspective. En P. Glotz, S. Bertschi \& C. Locke (Eds.), Tumb Culture. The meanings of mobile phones for society technology (pp. 23-35). Bielefeld: Transcript. 
Giddens, A. (2004). Consecuencias de la modernidad (tercera reimpresión). Madrid: Alianza Editorial.

Haddon, L. \& Silverstone, R. (1993). Teleworking in the 1990s. A view from the home. SPRU/CICT Report No. 10, University of Sussex. Recuperado en enero 9 de 2007 de la página Media@1se, del Departamento de Comunicación y Medios de la LSE.

Haddon, L. \& Silverstone, R. (1994). Lone parents and their information and Communications Technologies. SPRU/CICT Report, No. 12, University of Sussex, Recuperado en enero 9 de 2007, de la página Media@1se, del Departamento de Comunicación y Medios de la LSE.

Haddon, L. \& Silverstone, R. (1996). Information and Communication Technologies and the Young Elderly. Reporte ESRC/PICT. Recuperado enero 7 de 2007 de la página Media@1se, del Departamento de Comunicación y Medios de la LSE.

Haddon, L. (1998). The experience of the mobile phone. Presentado en 14 Congreso Mundial de Sociología. Montreal, julio 26-agosto 1, 1998. Recuperado en diciembre 1 de 2007 de la página Media@1se, del Departamento de Comunicación y Medios de la LSE.

Haddon, L. (2000). The social consequences of mobile telephony: Framing questions. Presentado en Sosiale Konsekvenser av Mobiltelefoni. Oslo, Noruega, junio 2000. Recuperado en diciembre 12 de 2007 de la página Media@1se, del Departamento de Comunicación y Medios de la LSE.

Haddon, L. (2003). Domestication and Mobile Technology. En J. E. Katz (Ed.), Machines that become us. The social context of personal communication technology (pp. 43-55). New Brunswick, N.J.: Transaction Publishers.

Haddon, L. (2006). Empirical studies using the domestication framework. En T. Berker, M. Hartmann, Y. Punie \& K. Ward (Eds.), Domestication of media and technology (pp. 103-122). Berkshire: Open University Press.

Haddon, L. (2007). Roger Silverstone's legacies: domestication. New media \& society 9 (1), 25-32.

Hartmann, M. (2006). The triple articulation of ITCs. Media as technological objects, symbolic environments and individual texts. En T. 
Berker, M. Hartmann, Y. Punie \& K. Ward (Eds.), Domestication of media and technology (pp. 80-102). Berkshire: Open University Press.

Helle-Valle, J. \& Slettemeås, D. (2008). ICTs, domestication and language-games: a Wittgensteinian approach to media uses. New media \& society, 10 (1), 45-66.

Höflich, J. (2004). A Certain Sense of Place. Mobile Communication and Local Orientation. Presentado en The Global and the Local in Mobile Communication. Places, Images, People, Connections. Budapest, Hungría, junio 10-12. Recuperado en octubre 3 de 2007 de la página de T-Mobile.

Höflich, J. (2005). The mobile phone and the dynamic between private and public communication: Results of an international exploratory study. En P. Glotz, S. Bertschi \& C. Locke (Eds.). Tumb Culture. The meanings of mobile phones for society technology (pp. 123135). Bielefeld: Transcript.

Humphreys, L. (2005). Cell phones in public: social interactions in a wireless era. New media \& society, 7 (6): 810-833.

Katz, E., Blumler, J. \& Gurevitch, M. (1973). Uses and gratifications research. The Public Opinion Quarterly, 37 (4): 509-523.

Katz, E., Blumler, J. \& Gurevitch, M. (1974). Utilization of mass communication by the individual. In J. G. Blumler \& E. Katz (Eds.), The uses of mass communications: Current perspectives on gratifications research (pp. 19-32). Beverly Hills: Sage.

Katz, J. E. \& Aakhus, M. (2002). Conclusion: Making meaning of mobiles -a theory of Apparatgeist. En J.E. Katz \& M. Aakhus (Eds.), Perpetual contact: Mobile communication, private talk, public performance (pp. 301-318). Cambridge UK: Cambridge University Press.

Katz, J. E. (2005). Mobile phones as fashion statements: The co-creation of mobile communication's public meaning. En R. Ling \& P. Pedersen (Eds.), Mobile communications: Re-negotiation of the social sphere (pp. 63-81). Surrey: Springer.

Katz, J. E. \& Sugiyama, S. (2006). Mobile phones as fashion statements: evidence from student surveys in the US and Japan. New media \& society, 8 (2), 321-337. 
Lacey, K. (2007). Home, work and everyday life: Roger Silverstone at Sussex. International Journal of Communication, 1, 61-69.

Lasen, A. (2005). Understanding mobile phone users and usage. Vodafone Group R\&D. Recuperado en octubre 1 de 2007 de la página de Vodafone.

Licoppe, C. \& Heurtin, J. (2002). Preserving the image. En J. E. Katz \& M. Aakhus (Eds.), Perpetual contact: Mobile communication, private talk, public performance (pp. 94-109). Cambridge UK: Cambridge University Press.

Ling, R. (1997). “One can talk about common manners!": The use of mobile telephones in inappropriate situations. En L. Haddon (Ed.), Themes in mobile telephony. Final Report COST 248. Recuperado en diciembre 2 de 2006 de la página del autor.

Ling, R. \& Helmersen, P. (2000). "It must be necessary, it has to cover a need": The adoption of mobile telephony. Presentado en Social Consequences of Mobile Telephony. Oslo, junio 16 del 2000. Recuperado en marzo 12 de 2007 de la página del autor.

Ling, R. (2001). The diffusion of mobile telephony among Norwegian teens: A report from after the revolution. Presentado en: ICUST. París, junio de 2001. Recuperado en mayo 4 de 2007 de la página del autor.

Ling, R. \& Yttri, B. (2002). Hyper-coordination via mobile phones in Norway. En J. E. Katz \& M. Aakhus (Eds.), Perpetual contact: Mobile communication, private talk, public performance (pp. 139169). Cambridge UK: Cambridge University Press.

Ling, R. (2004). The mobile connection. The cell phone's impact on society. San Francisco CA: Morgan Kaufmann Publishers.

Ling, R. (2008). The mediation of ritual interaction via the mobile telephone. En J. Katz (Ed.), Handbook of Mobile Communications Studies (pp.164-176). Cambridge, London: MIT Press.

Livingstone, S. (2007). On the material and the symbolic: Silverstone's double articulation of research traditions in new media studies. New media \& society, 9 (1), 16-24.

Morley, D. \& Silverstone, R. (1990). Domestic communication. Technologies and meanings. Media, culture \& society, 12, 31-55. 
Morley, D. (1992). Television, audiences and cultural studies. London: Routledge.

Morley, D. (1994). Comunicación y contexto. Perspectivas etnográficas sobre la audiencia de los medios. Versión, 4, 69-87.

Morley, D. (2006). Unanswered questions in audience research. The Communication Review, 9, 101-121.

Rogers, E. (1983). Diffusion of innovations. N.Y.: Free Press; London: Collier Macmillan.

Silverstone, R. (1991). From audiences to consumers: The households and the consumption of communication and information technologies. European Journal of Communication, 6, 135-154.

Silverstone, R. \& Hirsch, E. (1992). Consuming technologies. Media and information in domestic spaces. London: Routledge.

Silverstone, R., Hirsch, E. \& Morley, D. (1992). Information and communication technologies and the moral economy of the household. En R. Silverstone \& E. Hirsch (Eds.), Consuming technologies. Media and information in domestic spaces (pp. 15-31). London: Routledge.

Silverstone, R. (1993). Time, information and communication technologies and the household. Time and society, 2 (3), 283-311.

Silverstone, R. (1994). Television and Everyday Life. London: Routledge.

Silverstone, R. \& Haddon, L. (1996). Design and the domestication of information and communication technologies: Technical change and everyday life. En R. Silverstone \& R. Mansell (Eds.), Communication by design. The politics of information and communication technologies (pp. 44-74). Oxford: Oxford University Press.

Silverstone, R. (2002). Complicity and collusion in the mediation of everyday life. New Literary History, 33 (4), 761-780.

Silverstone, R. (2004). ¿Por qué estudiar los medios? Buenos Aires: Amorrortu Editores.

Silverstone, R. (2006). Domesticating domestication. Reflections on the life of a concept. En T. Berker, M. Hartmann, Y. Punie \& K. Ward (Eds.), Domestication of media and technology (pp. 229-248). Berkshire: Open University Press. 
Sørensen, K. (2006). Domestication: the enactment of technology. En T. Berker, M. Hartmann, Y. Punie \& K. Ward (Eds.), Domestication of media and technology (pp. 40-61). Berkshire: Open University Press.

Strathern, M. (1992). Foreword: The mirror of technololgy. En R. Silverstone \& E. Hirsch (Eds.), Consuming technologies. Media and information in domestic spaces (pp. VII-XII). London: Routledge. 